\title{
Exceso de peso y discapacidad en las personas mayores de la Argentina
}

\section{Excess weight and disability among the elderly in Argentina}

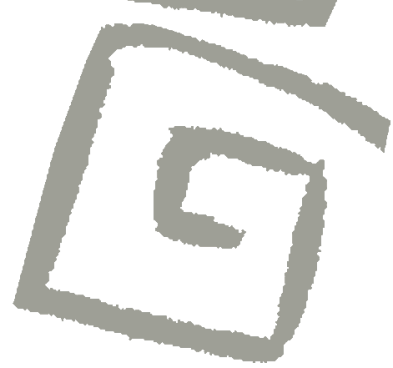

Monteverde, Malena'

${ }^{1}$ Doctora en Economía. Posdoctorada en Demografía. Investigadora Asistente, Centro de Investigaciones y Estudios sobre Cultura y Sociedad (CIECS), Universidad Nacional de Córdoba (UNC), CONICET, Argentina. montemale@yahoo.com
RESUMEN El objetivo del presente trabajo es analizar la relación entre el exceso de peso y la condición de discapacidad en las personas mayores de la Argentina y evaluar en qué medida podría estar operando algún factor protector que reduzca o atenúe el efecto del exceso de peso sobre la pérdida de capacidades funcionales en las personas mayores de 64 años. Para ello se utilizan los microdatos de la Encuesta Nacional de Factores de Riesgo 2009. Con el objeto de medir la asociación entre sobrepeso, obesidad y la condición de discapacidad, así como la interacción de la condición de peso y la edad, se estiman modelos de regresión logística. Los resultados indican que, si bien el sobrepeso y la obesidad tendrían un efecto neto positivo sobre la presencia de discapacidades, este sería menor entre las personas de 64 años y más. Los resultados sugieren que entre las personas mayores existe algún factor protector, que si bien no revierte la relación directa entre exceso de peso y discapacidad, parece atenuarla.

PALABRAS CLAVES Obesidad; Sobrepeso; Personas con Discapacidad; Adulto Mayor; Argentina.

\begin{abstract}
The aim of this paper is to analyze the relationship between excess weight and the condition of disability among elderly people in Argentina and to assess the extent to which a protective factor could be operating that reduces or mitigates the effect of overweight on the loss of functional skills in people over 64 years of age. In order to do so, microdata from Argentina's 2009 National Survey of Risk Factors [Encuesta Nacional de Factores de Riesgo] was utilized. To measure the association among overweight, obesity and disability status, as well as the interaction of weight status and age, logistic regression models were estimated. The results indicate that although overweight and obesity have a positive net effect on the occurrence of disabilities, this effect is lower among people 64 years of age and older. This result could be suggesting that among older people a protective factor is at work that, while not reversing the direct relationship between excess weight and disability, seems to attenuate it.
\end{abstract}

KEY WORDS Obesity; Overweight; Disabled Persons; Elderly; Argentina. 


\section{INTRODUCCIÓN}

La población de la Argentina se encuentra entre las más envejecidas de América Latina (junto con las de Uruguay y Cuba) y es de esperar que ello repercuta en el número de personas con discapacidad, ya que la probabilidad de presentar discapacidades se incrementa exponencialmente con la edad.

Sin embargo, si bien la edad es un factor de riesgo importante para aumentar la presencia de discapacidades, no es el único. Condiciones crónicas tales como enfermedades del corazón, cerebrovasculares, oseoarticulares, pulmonares, diabetes, pobres condiciones socioeconómicas padecidas a edades adultas o incluso durante la infancia tendrían un gran impacto en la probabilidad de reportar discapacidades en edades adultas y avanzadas (1-8).

Según estadísticas para el año 2000, la prevalencia de discapacidades en actividades de la vida diaria (AVD) entre las personas de 60 años y más de Buenos Aires (una de las regiones más envejecidas de la Argentina) era de más del 17,4\% y, tomando en cuenta las actividades instrumentales de la vida diaria (AIVD), dicha cifra ascendía a 27,1\%, casi un tercio de la población de esa edad (9).

En un estudio reciente en el que se comparan las prevalencias de discapacidad en AVD de grandes poblaciones urbanas de América Latina, se observa que las poblaciones más envejecidas no son las que presentan prevalencias más elevadas de autoreportes de discapacidades, pero sí se encontró una correlación entre los reportes de discapacidades y los reportes de condiciones crónicas altamente relacionadas con la discapacidad, sugiriendo que las diferencias no obedecerían simplemente a propensiones diferentes a reportar discapacidades entre las poblaciones (10).

Por otra parte, la obesidad es una condición que está alcanzando prevalencias de gran magnitud entre la población argentina. De acuerdo con estimaciones para el año 2010 de la Organización Mundial de la Salud (11), los hombres de 15 años y más de la Argentina presentarían una de las prevalencias de obesidad más altas de América (solo inferior a la de EE.UU.) alcanzando el 37,4\%. En el caso de las mujeres de esas edades dicha cifra sería más elevada $(37,8 \%)$, aunque en este caso la Argentina no se encuentra entre los primeros lugares del ranking ya que varios países presentan cifras aun más altas (entre ellos Barbados, Dominica, Trinidad y Tobago, EE.UU., Jamaica, Nicaragua, México, entre otros). En el estudio de Kain, Vio y Albala (12), Argentina ya aparece primera en el ranking de los países de América Latina con mayores prevalencias de obesidad en niños en edad preescolar sobre estadísticas para el año 1994.

Por su parte, una serie de estudios describen las particularidades de la obesidad en los países de la región: los países más pobres son los que presentan menores prevalencias de obesidad (13); en general, se observan mayores prevalencias de obesidad entre las mujeres en países con mayor ingreso per cápita y en aquellos con menores tasas de retraso en el crecimiento en los niños (13); se observan tendencias crecientes de obesidad en la medida en que los países emergen de la pobreza, en especial en áreas urbanas (14); entre los países de ingresos medios y en los más desarrollados la obesidad tiende a reducirse a medida que aumenta el ingreso, lo que resulta especialmente cierto entre las mujeres (15)

Un número creciente de trabajos (16-22) mencionan las deficiencias nutricionales tempranas, durante la gestación y la infancia, como un importante factor que incrementa el riesgo de obesidad en las poblaciones de la región, siguiendo la hipótesis de Barker (23). En tanto que los rápidos cambios nutricionales evidenciados en estos países, con incrementos en la ingesta de energía y en particular de grasas saturadas, y un estancamiento e incluso caída en el consumo de alimentos ricos en fibra, serían otro importante factor explicativo del aumento de la obesidad en la región (24). A lo anterior se sumarían los rápidos cambios en los patrones de actividad física, asociados con los procesos de urbanización y el cambio hacia trabajos y actividades recreativas más sedentarias $(18,25)$. Por otra parte, varios estudios específicos para la población Argentina muestran una clara asociación inversa entre obesidad y nivel socioeconómico (26-29) aunque dicha asociación no sería tan clara para el sobrepeso (9).

Respecto a la asociación entre obesidad y discapacidad existe una cierta controversia acerca de su efecto sobre la pérdida de capacidades funcionales, específicamente entre el grupo de personas 
de edades avanzadas. Por un lado, estudios a nivel internacional sugieren que la obesidad incrementa el riesgo de reportar discapacidades o dificulta la rehabilitación funcional $(29,30)$. Ello podría deberse en parte a que la condición de obesidad incrementa el riesgo de padecimiento de enfermedades altamente asociadas con la discapacidad como diabetes, enfermedades del corazón y enfermedades osteoarticulares (31-33), por lo que la relación entre obesidad y discapacidad parecería ser claramente positiva.

Sin embargo, hay estudios que sugieren que el exceso de peso podría tener un efecto protector en el grupo de personas mayores (33-37). El mecanismo protector sería especialmente importante entre las personas mayores, porque entre los principales factores de riesgo de discapacidad en este grupo de personas se encuentran las caídas y fracturas (menos presentes en las personas más jóvenes). Las fracturas estarían relacionadas con la presencia de osteoporosis, condición que a su vez parece ser menor entre las personas mayores con elevados niveles del índice de masa corporal (IMC) $(33-35,38)$. Por su parte, la reducción del riesgo de osteoporosis con el IMC podría deberse a la mayor producción de estrógenos del tejido adiposo y niveles de leptina más altas asociados al mayor peso corporal (39).

El estudio de Barrera et al. (33) se basa en una amplia muestra de personas de 70 años de edad y más pertenecientes a estratos socioeconómicos bajos, para los que se realizaron mediciones de la densidad mineral ósea y se compararon con los valores estándar correspondientes para determinar la presencia de osteoporosis. De acuerdo a los resultados, el riesgo de osteoporosis en hombres y mujeres con IMC mayor a $30 \mathrm{~kg} / \mathrm{m}^{2}$ es aproximadamente un 33\% menor comparado con los sujetos con IMC normal. En esta línea, el trabajo de Nakaoka et al. (34) encuentra que elevados niveles de IMC resultan protectores contra el riesgo de osteoporosis en hombres y mujeres de edades medias y de edades avanzadas. Wu et al. (35), basados en un estudio longitudinal, también encuentran una asociación entre cambios en el IMC y cambios en la densidad mineral ósea. Los anteriores hallazgos son consistentes con el resultado del trabajo de Kato et al. (36), quienes encuentran una menor tasa de fractura de cadera entre las personas obesas mayores de edad. Según Galvard et al. (37), la menor fractura también es consistente con la idea del "efecto de amortiguación" de la grasa corporal sobre las prominencias óseas reduciendo el impacto de potenciales caídas.

El objetivo del presente trabajo es analizar la relación entre el exceso de peso y la condición de discapacidad en las personas mayores de la Argentina y evaluar en qué medida podría estar operando algún factor protector que reduzca o atenúe el efecto de la obesidad sobre la pérdida de capacidades funcionales de las personas mayores.

\section{MATERIALES Y MÉTODOS}

El presente estudio se basa en un análisis transversal comparativo. Para medir discapacidad, obesidad y sobrepeso, así como la asociación entre la condición de peso (obesidad o sobrepeso) y la de discapacidad, se utilizaron los microdatos de la Encuesta Nacional de Factores de Riesgo (ENFR) de 2009, que provee información autoreportada de la población de 18 años y más de la Argentina sobre: talla, peso, padecimiento de enfermedades crónicas, problemas de movilidad, dificultades para el cuidado personal y para la realización de actividades cotidianas, además de un conjunto de variables sociodemográficas como nivel de educación, ingresos, edad y sexo.

La ENFR es una encuesta de corte transversal llevada a cabo entre octubre, noviembre y diciembre del año 2009 por el Ministerio de Salud de la Nación y el Instituto Nacional de Estadística y Censos de Argentina, con la colaboración de la direcciones provinciales de estadística. Los objetivos de la encuesta fueron:

- conocer la distribución de los factores de riesgo en la población de 18 años y más;

- estimar su prevalencia;

- determinar el perfil de la población bajo riesgo a través de sus características sociodemográficas, socioeconómicas, educativas y del entorno familiar social. (40 p.5)

La muestra final de la ENFR 2009 contó con información para 34.732 individuos de 18 años y más, de los cuales 5.751 eran mayores de 64 años (40). Extrapolando los datos de la muestra a 
la población se obtiene una media de edad en el grupo de 18 a 64 años de 38 años (39 años para la muestra sin extrapolar) y una proporción de mujeres del 53\% (56\% sin extrapolar). Por su parte, en el grupo de 65 años y más la media de edad es de 74 años (igual valor sin extrapolar) y un $57 \%$ son mujeres (62\% sin extrapolar).

En cuanto a la distribución geográfica de la población, la región del Gran Buenos Aires (Ciudad Autónoma de Buenos Aires y 24 partidos de la provincia de Buenos Aires que rodean a la Ciudad) y la región Pampeana (provincia de Buenos Aires -excluyendo los 24 partidos del Conurbano Bonaerense-, Córdoba, La Pampa, Santa Fe y Entre Ríos) concentran más del $70 \%$ de la población de 18 a 64 años y el $77 \%$ de la población mayor de 64 años (valores extrapolados).

Para identificar a las personas con alguna discapacidad se consideraron las tres condiciones contempladas en la encuesta. Para ello, se construyó una variable dicotómica con valor igual a uno cuando la persona entrevistada responde:

- tener problemas de movilidad (cuando responde que tiene problemas para caminar o tiene que estar en la cama);

- problemas con el cuidado personal (cuando la persona responde que tiene problemas o no puede vestirse y lavarse sola);

- problemas con las actividades cotidianas (cuando responde tener problemas o ser incapaz de realizar dichas actividades).

Es decir, la persona se considera "discapacitada" cuando presenta al menos una de las tres condiciones antes mencionadas.

Para clasificar a la población según peso se tomaron categorías de IMC ampliamente utilizadas para la población adulta no asiática $(41,42)$, según las cuales los individuos con IMC $<18,5$ pertenecen a la categoría "bajo peso", aquellos con IMC entre 18,5 y 24,99 a la categoría "peso normal", con IMC $\geq 25$ a la de "sobrepeso" y con IMC $\geq 30$ a la de "obeso". Sobre la base de las anteriores definiciones se creó una variable dicotómica para cada una de las categorías de peso.

Se calcularon prevalencias de discapacidad y de peso, en la muestra y en la población representada, y se estimaron los respectivos intervalos de confianza asumiendo un diseño de muestreo simple al azar, debido a que no se cuenta con la información del diseño para la correcta estimación de las varianzas en la base de la ENFR. Esta restricción para el cálculo de los intervalos de confianza introduce inconsistencias con los valores de las prevalencias ponderadas, dado que estas sí tienen en cuenta la información de diseño complejo. Por lo tanto, la comparación de las prevalencias ponderadas y los intervalos de confianza debe realizarse con cautela, siendo más apropiada la comparación con las prevalencias sin ponderar (que asumen implícitamente un diseño de muestro simple al azar).

Por otra parte, para el cálculo de los intervalos de confianza se aplicó el método exacto basado en la distribución $F$, dado que para la distribución binomial resulta un procedimiento superior a otras aproximaciones como las basadas en el teorema central del límite en la distribución de Poisson (43). Además, para medir la asociación entre el exceso de peso y la discapacidad y evaluar en qué medida dicha asociación es diferente entre las personas mayores de 64 años, se realizaron los siguientes cálculos:

1. Se estimó la prevalencia de discapacidad condicionada a cada una de las categorías de peso para las personas de 18 a 64 años por un lado, $y$ para las personas mayores de 64 años por otro.

2. Se estimaron modelos logísticos con la variable dependiente "discapacidad" (variable dicotómica construida según se detalló anteriormente) controlando por posibles efectos de confusión, como pueden ser el sexo y el nivel de instrucción de los individuos.

a. Primero se estimaron los modelos separados para cada grupo de edad, con el fin de evaluar la asociación entre las variables explicativas y la condición de discapacidad en cada grupo.

b. Luego se estimó un modelo para todas las edades en el que se incluyeron términos de interacción entre las categorías de peso y los grupos de edad con el fin de evaluar específicamente si las diferencias de asociación entre la condición de obesidad y de discapacidad entre los grupos de edad resultaban estadísticamente significativas. 
Todos los cálculos, excepto los intervalos de confianza de las prevalencias incluidos en los Cuadros 1, 2 y 3 han sido realizados con el programa STATA/SE, versión 12 (StataCorp LP). La estimación robusta de los errores estándar de los modelos logísticos corresponde al Ilamado "Huber Sandwich Estimator" el cual ayuda a mejorar la estimación de la varianza de estimador de máxima verosimilitud cuando el modelo subyacente no es aproximadamente el correcto (44). Los intervalos de confianza de los Cuadros 1, 2 y 3 fueron calculados utilizando el programa Excel de acuerdo a las expresiones del trabajo de Correa y Sierra (43).

\section{RESULTADOS}

Los cuadros de resultados que se presentan a continuación contienen el número de personas totales, las prevalencias y sus respectivos intervalos de confianza de las condiciones de discapacidad y de peso, entre la población adulta de 18 a 64 años y la población mayor de 64 años, con el fin de evaluar el patrón de discapacidades y de categorías de peso de los dos grupos de edad.
En el Cuadro 1 se presentan los resultados para las condiciones de discapacidad. Los totales expandidos a la población muestran que más de 1,8 millones de personas de 18 a 64 años y casi 1,4 millones de personas mayores de 64 años tendrían al menos una de las tres condiciones de discapacidad consideradas, siendo las más frecuentes las dificultades para caminar (en ambos grupos de edad).

Si bien en valores absolutos el número de personas con alguna discapacidad sería mayor entre los más jóvenes, en términos relativos este resultado se invierte, ya que el número total de personas mayores (con y sin discapacidades) es sustancialmente más bajo. Así, la prevalencia de "alguna de las tres discapacidades" sería más de 4 veces superior en el grupo de adultos mayores (afectando al 37\% de la población de 65 años y más) respecto a la observada entre la población de 18 a 64 años de edad (del orden del 9\%).

La prevalencia de discapacidad autoreportadas asociadas con dificultades para caminar alcanzarían casi el 7\% en la población de 18 a 64 años y más del 33\% entre la población de 65 años y más. Por su parte, las dificultades para la realización de actividades cotidianas se presentarían en un $5 \%$ de la población de 18 a 64 años y en el

Cuadro 1. Prevalencia de discapacidades, según grupo de edad y tipo de discapacidad. Argentina, 2009.

\begin{tabular}{|c|c|c|c|c|c|}
\hline $\begin{array}{l}\text { Discapacidad según } \\
\text { grupos de edad }\end{array}$ & $\begin{array}{c}\text { Muestra } \\
\text { expandida } \\
(\mathrm{n}=\mathbf{2 4 . 4 3 4 . 5 9 5 )}\end{array}$ & $\begin{array}{c}\text { Muestra } \\
(\mathrm{n}=34.732)\end{array}$ & $\begin{array}{c}\text { Prevalencia } \\
\text { ponderada } \\
\text { (por 100) }\end{array}$ & $\begin{array}{l}\text { Prevalencia } \\
\text { sin ponderar } \\
\text { (por 100) }\end{array}$ & IC95\% ${ }^{1}$ \\
\hline \multicolumn{6}{|l|}{18 A 64 AÑOS } \\
\hline Caminar & 1.422 .385 & 2.102 & 6,87 & 7,25 & 7,$25 ; 7,25$ \\
\hline Cuidado personal & 264.734 & 401 & 1,28 & 1,38 & 1,$38 ; 1,39$ \\
\hline Actividades cotidianas & 1.038 .636 & 1.587 & 5,02 & 5,48 & 5,$48 ; 5,48$ \\
\hline Alguna discapacidad ${ }^{2}$ & 1.870 .153 & 2.799 & 9,03 & 9,66 & 9,$66 ; 9,66$ \\
\hline \multicolumn{6}{|l|}{65 AÑOS Y MÁS } \\
\hline Caminar & 1.245 .133 & 2.028 & 33,35 & 35,26 & 35,$26 ; 35,27$ \\
\hline Cuidado personal & 293.133 & 530 & 7,85 & 9,22 & 9,$21 ; 9,23$ \\
\hline Actividades cotidianas & 772.301 & 1.295 & 20,69 & 22,52 & 22,$51 ; 22,53$ \\
\hline Alguna discapacidad ${ }^{2}$ & 1.390 .356 & 2.274 & 37,24 & 39,54 & 39,$53 ; 39,55$ \\
\hline
\end{tabular}

Fuente: Elaboración propia a partir de la base de microdatos de la Encuesta Nacional de Factores de Riesgo 2009.

${ }^{1}$ Los intervalos de confianza se estimaron a través del método exacto, asumiendo un diseño de muestreo simple al azar.

${ }^{2}$ Los valores expresados en "alguna discapacidad" corresponden a corresponden a los individuos con problemas de movilidad, problemas con el cuidado personal o problemas con las actividades cotidianas. 
Cuadro 2. Prevalencia de estado nutricional, según grupo de edad y categoría de peso. Argentina, 2009 .

\begin{tabular}{|c|c|c|c|c|c|}
\hline $\begin{array}{l}\text { Categoría de peso } \\
\text { según grupos de edad }\end{array}$ & $\begin{array}{c}\text { Muestra } \\
\text { expandida } \\
(\mathrm{n}=\mathbf{2 3 . 1 4 4 . 6 8 0 )}\end{array}$ & $\begin{array}{c}\text { Muestra } \\
(\mathrm{n}=32.448)\end{array}$ & $\begin{array}{c}\text { Prevalencia } \\
\text { ponderada } \\
\text { (por 100) }\end{array}$ & $\begin{array}{l}\text { Prevalencia } \\
\text { sin ponderar } \\
\text { (por 100) }\end{array}$ & $\mathrm{IC} 5 \%^{1}$ \\
\hline \multicolumn{6}{|l|}{18 A 64 AÑOS } \\
\hline Obesidad & 3.472 .867 & 4.969 & 17,65 & 18,23 & 18,$23 ; 18,23$ \\
\hline Sobrepeso & 6.710 .959 & 9.592 & 34,10 & 35,19 & 35,$19 ; 35,19$ \\
\hline Normal & 8.974 .889 & 12.065 & 45,61 & 44,27 & 44,$26 ; 44,27$ \\
\hline Bajo peso & 518.017 & 630 & 2,63 & 2,31 & 2,$31 ; 2,32$ \\
\hline \multicolumn{6}{|l|}{65 AÑOS Y MÁS } \\
\hline Obesidad & 696.856 & 1.049 & 20,09 & 20,20 & 20,$20 ; 20,22$ \\
\hline Sobrepeso & 1.493 .533 & 2.189 & 43,07 & 42,16 & 42,$15 ; 42,17$ \\
\hline Normal & 1.237 .783 & 1.888 & 35,69 & 36,36 & 36,$36 ; 36,38$ \\
\hline Bajo peso & 39.776 & 66 & 1,15 & 1,27 & 1,$27 ; 1,29$ \\
\hline
\end{tabular}

Fuente: Elaboración propia a partir de la base de microdatos de la Encuesta Nacional de Factores de Riesgo 2009.

${ }^{1}$ Los intervalos de confianza se estimaron a través del método exacto, asumiendo un diseño de muestreo simple al azar.

$21 \%$ de los adultos mayores; mientras que la prevalencia de discapacidad asociada con el cuidado personal afectaría al $1 \%$ de la población más joven y a casi el $8 \%$ de la población mayor.

El Cuadro 2 muestra los resultados para las cuatro categorías de peso (obesidad, sobrepeso, peso normal y bajo peso) para los dos grupos de edad. Entre los mayores de 64 años se observan prevalencias de sobrepeso y obesidad superiores a las observadas en el grupo de 18 a 64 años (63\% versus $52 \%$ ), aun cuando en ambos casos superan el $50 \%$ de la población. Esto da cuenta de la elevada prevalencia de sobrepeso y obesidad en todas las edades y de la asociación positiva entre el IMC y la edad.

Por otra parte, la magnitud de las diferencias entre los grupos etarios analizados marca mayor distancia en el sobrepeso que en la obesidad. Es así como el sobrepeso entre las personas de 18 a 64 años y las mayores de 64 años sube del 34\% al $43 \%$, respectivamente; mientras que la prevalencia de obesidad asciende del $18 \%$ al $20 \%$ en cada grupo de edad, respectivamente. Dichas prevalencias implican que, entre la población más joven, habría 3,5 millones de personas obesas y 6,7 millones de personas con sobrepeso en la
Argentina; mientras que, entre los mayores de 64 años, esas cifras ascienden a más de 696 mil personas obesas y casi 1,5 millones con sobrepeso.

En el Cuadro 3 se puede observar la prevalencia de discapacidad en cada categoría de peso. Las prevalencias de discapacidad entre las personas con sobrepeso y obesidad resultan superiores a las observadas en las personas con peso normal, tanto entre las personas de 18 a 64 años, como entre los mayores de 64 años.

Entre los más jóvenes la prevalencia de discapacidad alcanza al 9\% de las personas con sobrepeso y a casi el $16 \%$ de las personas con obesidad; mientras que, entre los jóvenes con peso normal, la prevalencia de discapacidad es del $6 \%$. Estas diferencias implican que, en este grupo etario, la discapacidad sería un $50 \%$ más en las personas con sobrepeso, respecto de las de peso normal. Asimismo, las personas obesas presentan un exceso de prevalencia de discapacidad de más del $160 \%$ (respecto de las de peso normal).

Por su parte, dichos "excesos de discapacidad" resultan ser mucho menores en el grupo de mayores de 64 años (del 1\% y del 30\% entre sobrepeso y obesidad, respectivamente), aun cuando las prevalencias de discapacidad son 
mucho mayores en este grupo etario, dado que entre las personas con sobrepeso alcanzan el 34\% y con obesidad el $44 \%$. Además, resulta importante señalar que un tercio de las personas con peso normal presentan alguna de las dificultades incapacitantes que se han analizado. El resultado anterior sugiere que si bien el exceso de peso se asocia con una mayor prevalencia en la presencia de discapacidades tanto en la población adulta como en la mayor de 64 años, entre esta última podrían existir factores atenuantes de la relación (consistentes con la teoría de efecto protector).

Para evaluar en qué medida este resultado se mantiene, es importante medir la relación controlando por factores de composición demográfica propios de cada grupo de edad (por ejemplo, entre las personas mayores hay más mujeres) y por factores socioeconómicos (por ejemplo, diferencias de composición por niveles de instrucción).

El Cuadro 4 muestra los resultados del modelo logístico de la probabilidad de reportar discapacidad (al menos una de las tres contempladas en la encuesta) para cada grupo de edad por separado.

El odds ratio del sobrepeso y la obesidad resulta mayor en el primer grupo etario (18 a 64 años) de forma coincidente con los resultados anteriores. En el grupo de 18 a 64 años la chance de reportar discapacidades entre las personas con sobrepeso es un $42 \%$ superior que entre las personas con peso normal; mientras que, en el grupo de mayores de 64 años, si bien el odds ratio es algo mayor a uno, no es estadísticamente significativo. Por su parte, entre las personas más jóvenes la chance de reportar discapacidades entre las personas obesas es un $135 \%$ superior a las de peso normal, mientras que entre los mayores de 64 años dicha chance es del 57\%.

Otras asociaciones que surgen del modelo indican que entre las mujeres de ambos grupos etarios hay una mayor chance de reportar discapacidades, y que entre la población con estudios formales (ya sean estudios primarios, secundarios o terciarios incompletos o más) la chance de reportar discapacidades es menor respecto a la población sin instrucción (grupo de referencia) y ello es cierto tanto en el grupo de 18 a 64 años como para los mayores de dicha edad.

El Cuadro 5 muestra los resultados del modelo logístico para la probabilidad de reportar discapacidades en las personas de 18 años y más (totales)

Cuadro 3. Prevalencia de discapacidad, según estado nutricional y grupos de edad. Argentina, 2009.

\begin{tabular}{lccccc}
\hline $\begin{array}{l}\text { Categoría de peso } \\
\text { según grupo de } \\
\text { edad }\end{array}$ & $\begin{array}{c}\text { Muestra } \\
\text { expandida } \\
(\mathbf{n = 2 . 9 8 1 . 4 5 9 )}\end{array}$ & $\begin{array}{c}\text { Muestra } \\
(\mathbf{n}=\mathbf{4 . 5 3 3 )}\end{array}$ & $\begin{array}{c}\text { Prevalencia } \\
\text { ponderada } \\
\text { (por 100) }\end{array}$ & $\begin{array}{c}\text { Prevalencia } \\
\text { sin ponderar } \\
\text { (por 100) }\end{array}$ & IC95\% $^{\mathbf{1}}$ \\
\hline 18 A 64 AÑos & & & & & \\
$\quad$ Obesidad & 550.221 & 807 & 15,84 & 16,24 & 16,$24 ; 16,26$ \\
Sobrepeso & 610.023 & 906 & 9,09 & 9,45 & 9,$44 ; 9,45$ \\
Normal & 543.097 & 802 & 6,05 & 6,65 & 6,$65 ; 6,66$ \\
Bajo peso & 24.312 & 39 & 4,69 & 6,19 & 6,$18 ; 6,34$ \\
65 AÑos Y MÁS & & & & & \\
Obesidad & 304.384 & 487 & 43,68 & 46,43 & 46,$38 ; 46,48$ \\
Sobrepeso & 510.445 & 791 & 34,18 & 36,14 & 36,$12 ; 36,16$ \\
Normal & 417.116 & 672 & 33,70 & 35,59 & 35,$57 ; 35,63$ \\
Bajo peso & 21.861 & 29 & 54,96 & 43,94 & 43,$28 ; 44,78$
\end{tabular}

Fuente: Elaboración propia a partir de la base de microdatos de la Encuesta Nacional de Factores de Riesgo 2009.

Nota: El total de la población con discapacidad y total de población en esta tabla resultan menores porque solo se consideran los casos para los cuales hay información sobre la categoría de peso.

${ }^{1}$ Los intervalos de confianza se estimaron a través del método exacto, asumiendo un diseño de muestreo simple al azar. 
Cuadro 4. Regresión logística para la asociación entre discapacidad y variables predictoras seleccionadas, según grupos etarios. Argentina, 2009.

\begin{tabular}{|c|c|c|c|c|c|}
\hline Variables & OR & EER & $\mathrm{z}$ & $\mathbf{P}>\mathbf{z}$ & IC95\% \\
\hline \multicolumn{6}{|l|}{18 A 64 AÑOS } \\
\hline \multicolumn{6}{|c|}{ CATEGORÍA DE PESO } \\
\hline Obesidad & 2,353 & 0,131 & 15,360 & 0,000 & 2,$110 ; 2,625$ \\
\hline Sobrepeso & 1,418 & 0,075 & 6,650 & 0,000 & 1,$279 ; 1,572$ \\
\hline Normal $^{1}$ & - & - & - & - & - \\
\hline Bajo peso & 0,860 & 0,149 & $-0,870$ & 0,383 & 0,$613 ; 1,207$ \\
\hline \multicolumn{6}{|l|}{ SEXO } \\
\hline Varón ${ }^{1}$ & - & - & - & - & - \\
\hline Mujer & 1,593 & 0,071 & 10,410 & 0,000 & 1,$460 ; 1,740$ \\
\hline \multicolumn{6}{|l|}{ NIVEL EDUCATIVO } \\
\hline Sin instrucción ${ }^{1}$ & - & - & - & - & - \\
\hline Primaria & 0,478 & 0,066 & $-5,350$ & 0,000 & 0,$365 ; 0,627$ \\
\hline Secundaria & 0,207 & 0,029 & $-11,350$ & 0,000 & 0,$157 ; 0,271$ \\
\hline Terciario & 0,136 & 0,020 & $-13,840$ & 0,000 & 0,$102 ; 0,180$ \\
\hline \multicolumn{6}{|l|}{65 AÑOS Y MÁS } \\
\hline \multicolumn{6}{|c|}{ CATEGORÍA DE PESO } \\
\hline Obesidad & 1,567 & 0,125 & 5,620 & 0,000 & 1,$340 ; 1,832$ \\
\hline Sobrepeso & 1,065 & 0,071 & 0,940 & 0,347 & 0,$934 ; 1,214$ \\
\hline Normal $^{1}$ & - & - & - & - & - \\
\hline Bajo peso & 1,204 & 0,306 & 0,730 & 0,466 & 0,$731 ; 1,982$ \\
\hline \multicolumn{6}{|l|}{ SEXO } \\
\hline Varón ${ }^{1}$ & - & - & - & - & \\
\hline Mujer & 1,579 & 0,096 & 7,510 & 0,000 & 1,$402 ; 1,779$ \\
\hline \multicolumn{6}{|l|}{ NIVEL EDUCATIVO } \\
\hline Sin instrucción ${ }^{1}$ & - & - & - & - & - \\
\hline Primaria & 0,610 & 0,081 & $-3,700$ & 0,000 & 0,$470 ; 0,793$ \\
\hline Secundaria & 0,374 & 0,054 & $-6,830$ & 0,000 & 0,$282 ; 0,496$ \\
\hline Terciario & 0,309 & 0,049 & $-7,410$ & 0,000 & 0,$226 ; 0,421$ \\
\hline
\end{tabular}

Fuente: Elaboración propia a partir la base de microdatos de la Encuesta Nacional de Factores de Riesgo 2009.

${ }^{1}$ Valor de referencia. OR=Odds ratio; EER= Error estándar robusto; IC95\%=Intervalo de confianza al 95\%.

con los términos de interacción de interés, con el fin de evaluar específicamente si las interacciones entre las condiciones de exceso de peso y el grupo de edad son estadísticamente significativas.

Dado que, para los tres niveles de instrucción formal, la asociación con las chances de reportar discapacidad es negativa (y estadísticamente significativa), en el siguiente modelo se reagruparon las categorías en dos: con y $\sin$ instrucción. Cabe destacar que los odds ratio de los términos de interacción entre la condición de obesidad y el grupo de edad son ambos mayores a uno (siendo que el grupo de referencia en cada caso son las personas de peso normal), el odds ratio es más elevado para el término de interacción de los obesos más jóvenes (comparado con el de los obesos mayores a 64 años) y las diferencias entre los dos términos de interacción (para los obesos jóvenes y los obesos mayores de edad) son estadísticamente significativas ya que 
Cuadro 5. Regresión logística para la asociación entre discapacidad y variables predictoras con inclusión de términos de interacción. Argentina, 2009.

\begin{tabular}{|c|c|c|c|c|c|}
\hline Variables & OR & EER & $\mathbf{z}$ & $\mathrm{P}>\mathrm{z}$ & IC95\% \\
\hline \multicolumn{6}{|c|}{ CATEGORÍA DE PESO SEGÚN EDAD } \\
\hline \multicolumn{6}{|l|}{18 A 64 AÑos } \\
\hline Obesidad & 1,615 & 0,128 & 6,030 & 0,000 & 1,$382 ; 1,887$ \\
\hline Sobrepeso & 1,074 & 0,072 & 1,070 & 0,285 & 0,$942 ; 1,224$ \\
\hline Normal $^{1}$ & - & - & - & - & - \\
\hline Bajo peso & 0,836 & 0,143 & $-1,050$ & 0,296 & 0,$597 ; 1,170$ \\
\hline \multicolumn{6}{|l|}{65 AÑOS Y MÁS } \\
\hline Obesidad & 2,870 & 0,155 & 19,550 & 0,000 & 2,$582 ; 3,190$ \\
\hline Sobrepeso & 1,588 & 0,082 & 8,970 & 0,000 & 1,$436 ; 1,758$ \\
\hline Normal $^{1}$ & - & - & - & - & - \\
\hline Bajo peso & 1,267 & 0,321 & 0,930 & 0,350 & 0,$771 ; 2,083$ \\
\hline \multicolumn{6}{|l|}{ EDAD } \\
\hline 18 a 64 años $^{1}$ & - & - & - & - & - \\
\hline 65 años y más & 7,543 & 0,465 & 32,790 & 0,000 & 6,$685 ; 8,511$ \\
\hline \multicolumn{6}{|l|}{ SEXo } \\
\hline Varón ${ }^{1}$ & - & - & - & - & - \\
\hline Mujer & 1,562 & 0,056 & 12,370 & 0,000 & 1,$455 ; 1,676$ \\
\hline \multicolumn{6}{|l|}{ NIVEL EDUCATIVO } \\
\hline Sin instrucción ${ }^{1}$ & - & - & - & - & 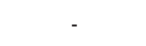 \\
\hline Con instrucción & 0,367 & 0,039 & $-9,480$ & 0,000 & 0,$298 ; 0,451$ \\
\hline
\end{tabular}

Fuente: Elaboración propia a partir la base de microdatos de la Encuesta Nacional de Factores de Riesgo 2009.

${ }^{1}$ Valor de referencia. OR= Odds ratio; EER= Error estándar robusto; IC95\%=Intervalo de confianza al 95\%.

el coeficiente estimado de cada término no se incluye en el intervalo de confianza del otro, sugiriendo que, en efecto, el incremento en la chance de reportar discapacidades en el grupo de 64 años obesos respecto de los de peso normal es menor que el mismo incremento medido en la población de 18 a 64 años. Por su parte, los términos de interacción entre el grupo de edad y el sobrepeso también resultan mayores a uno (aunque solo es estadísticamente significativa para los menores de 65 años) y diferentes entre sí, lo cual también resulta consistente con la idea de un menor incremento en la chance de reportar discapacidades entre las personas mayores de edad con sobrepeso respecto de las de peso normal que el mismo incremento en las personas más jóvenes.

\section{DISCUSIÓN}

Dada la importancia de la discapacidad asociada al proceso de envejecimiento poblacional en la Argentina y a la creciente obesidad y exceso de peso entre la población de todos los grupos etarios de este país, resulta de interés analizar la asociación entre estas dos condiciones. El exceso de peso es una condición que incrementa el riesgo de padecimiento de condiciones crónicas tales como enfermedades del corazón, cerebrovasculares, osteoarticulares, pulmonares, diabetes, las que a su vez tendrían un importante efecto en la probabilidad de reportar discapacidades en edades adultas y avanzadas. 
En este sentido, la relación entre el exceso de peso y la discapacidad parece ser claramente positiva y diversos estudios han arrojado evidencia sobre esto $(29,30)$. Sin embargo, otro cuerpo de bibliografía arroja evidencias de la existencia de un factor protector del exceso de peso sobre el riesgo de discapacidad, al menos en personas adultas y mayores (33-37). Dicho factor parecería estar dado por el menor riesgo de osteoporosis observado entre las personas de mayor edad con IMC superior a $30 \mathrm{~kg} / \mathrm{m}^{2}(33,34)$, lo cual reduce el riesgo de fracturas y con ello las consecuencias de las caídas sobre el riesgo de discapacidad de las personas mayores $(36,37)$.

Los resultados de este trabajo muestran prevalencias de discapacidad y de obesidad más elevadas para las personas de mayor edad (65 años y más), respecto al grupo más joven (18 a 64 años), consistentemente con los hallazgos de estudios sobre obesidad y discapacidad por separado $(45,46)$. Por otra parte, también se observa un patrón inverso entre la condición de discapacidad y el nivel de educación, en línea con los resultados obtenidos en trabajos sobre los principales factores de riesgo y de "protección" de la condición de discapacidad $(9,10)$. Además, las mujeres presentan una mayor probabilidad de reportar discapacidades aun controlando por la edad, resultado que está ampliamente documentado en la bibliografía sobre el tema $(9,10)$.

El principal resultado de este estudio muestra que el incremento en la chance de reportar discapacidad en el grupo de población de mayores de 64 años obesos o con sobrepeso respecto a los de peso normal es menor que el mismo incremento en la población de 18 a 64 años, lo cual podría estar sugiriendo que efectivamente entre las personas mayores estaría operando un factor protector del exceso de peso (sobrepeso u obesidad), que si bien no revierte la relación directa entre exceso de peso y discapacidad, parece atenuarla.

Sin embargo, entre las principales limitaciones de este estudio se encuentra el hecho de que las medidas de discapacidad y obesidad son autoreportadas. Los autoreportes de salud son ampliamente utilizados, en especial en estudios a nivel poblacional en los que difícilmente se cuenta con mediciones directas de las condiciones (10), y si bien pueden presentar imprecisiones, suelen ser buenas aproximaciones de lo que se busca medir
$(8,45,46)$. Por otra parte, si bien los autoreportes pueden presentar algún grado de sesgo, no hay razones para pensar que afecten la relación entre las dos condiciones y menos aún las diferencias según los grupos etarios.

Un aspecto que sí podría implicar un sesgo relevante en el objeto de estudio de este trabajo es la utilización del IMC para medir obesidad en los diferentes grupos de población. El IMC es una medida aproximada del exceso de peso y la composición corporal en todos los grupos de edad y existen dudas respecto de que pueda ser usada para comparar prevalencias de obesidad, especialmente entre diferentes grupos etarios.

En el caso específico de las personas mayores, que suelen tener una pérdida de altura como consecuencia de la compresión vertebral que se produce con la edad, un IMC superior a 30 podría representar una menor proporción de grasa corporal que la misma medida en la misma persona varios años antes (47-50). Si lo anterior es cierto, en el grupo de personas mayores con sobrepeso u obesidad se estaría incluyendo a personas con peso normal, para las cuales la relación con el riesgo de discapacidad es menor (para todas las edades). Por lo tanto, la menor asociación entre el exceso de peso y la discapacidad en este grupo etario podría ser producto de una sobreestimación del sobrepeso y la obesidad y no de la existencia de un factor protector.

Por otra parte, cabe indicar que dada la naturaleza de los datos con los que se cuenta (de corte transversal) no ha sido posible descontar de la relación entre el exceso de peso y la discapacidad el efecto debido a la "causalidad reversa", que es esperable que exista en alguna medida, ya que los individuos con alguna discapacidad tienden a realizar actividad física en menor medida que los individuos sin dificultades funcionales. Si bien lo anterior es cierto, para que la causalidad reversa afecte las conclusiones de este estudio, dicho efecto debería ser de una magnitud significativamente diferente en los dos grupos de edad analizados.

Para poder avanzar en el estudio de la relación entre obesidad y discapacidad resulta fundamental contar con mejores mediciones de las dos condiciones. En especial, y por lo mencionado anteriormente, se necesitarían medidas adicionales que sean más precisas en la diferenciación de los componentes corporales, más allá 
de la valoración de corpulencia que estima el IMC en todos los grupos etarios e, idealmente, contar con datos de corte longitudinal que permitan identificar la causalidad de la relación entre el exceso de peso y la condición de discapacidad.

Los resultados de este estudio ponen de relieve la necesidad de avanzar en líneas de investigación que permitan profundizar el conocimiento de la relación entre el exceso de peso y la discapacidad, y la importancia de contemplar las particularidades de los distintos grupos de población, de forma tal que sirvan de base para el diseño de políticas de salud pública.

\section{AGRADECIMIENTOS}

Se agradece profundamente a los evaluadores y a los editores de la revista Salud Colectiva por sus comentarios y sugerencias, que han sido de gran importancia para la mejora de la versión original del trabajo.

\section{REFERENCIAS BIBLIOGRÁFICAS}

1. Verbrugge LM, Lepkowski JM, Imanaka Y. Comorbidity and its impact on disability. The Milbank Quarterly. 1989;67(3/4):450-484.

2. Elo I, Preston S. Effects of early-life conditions on adult mortality: a review. Population Index. 1992;58(2):186-212.

3. Barker D. Mothers, babies and health in later life. Second Edition. London: Churchill Livingstone; 1998.

4. Fried LP, Bandeen-Roche K, Kasper JD, Guralnik JM. Association of comorbidity with disability in older women: The women's health and aging study. Journal of Clinical Epidemiology. 1999;52(1):27-37.

5. Couzin J. Quirks of fetal environment felt decades later. Science. 2002;296(5576):2167-2169.

6. Palloni A, McEniry M, Davila AL, García-Gurucharri A. The influence of early conditions on health status among elderly Puerto Ricans. Social Biology. 2005;52(3-4):132-163.

7. Kuh D, Hardy R, Butterworth S, Okell L, Richards M, Wadsworth M, Cooper C, Sayer AA. Developmental origins of midlife physical performance: evidence from a British birth cohort. American Journal of Epidemiology. 2006;164(2):110-121.

8. Guralnik JM, Butterworth S, Wadsworth MEJ, Kuh D. Childhood socioeconomic status predicts physical functioning a half century later. Journals of Gerontology Series A, Biological Sciences and Medical Sciences. 2006;61(7):694-701.
9. Monteverde M, Pelaez E, Celton D. Level of educaction and disability among the elderly from Buenos Aires. Población y Salud en Mesoamérica. 2012;10(1):1-17.

10. Monteverde-Verdenelli M, Peranovich-Celton A, Zepeda-Ortega A. Comparación de la prevalencia de discapacidades basada en autoreportes en países de América Latina. Población y Salud en Mesoamérica. 2014;12(1):1-11.

11. World Health Organization. WHO Global Infobase [Internet]. 20 ene 2011 [citado 14 feb 2014]. Disponible en: https://goo.gl/poRWpg.

12. Kain J, Vio F, Albala C. Obesity trends and determinant factors in Latin America. Cadernos de Saúde Pública. 2003;19(Supl 1):S77-S86.

13. Martorell $M$, Kettel-Khan $L$, Hughes $M$, Grummer-Strawn L. Obesity in Latin American women and children. Journal of Nutrition. 1998;128:1464-1473.

14. Peña $M$, Bacallao J. Obesity and poverty a new public health change (Scientific Publication 576). Washington DC: PAHO; 2000.

15. Aranceta J, Perez-Rodrigo C, Serra-Majem L, Ribas L, Quiles-Izquierdo J, Vioque J, Foz M, Spanish Collaborative Group for the Study of Obesity. Influence of sociodemographic factors in the prevalence of obesity in Spain. The SEEDO'97 study. European Journal of Clinical Nutrition. 2001;55:430-435.

16. Popkin B, Richards M, Monteiro C. Stunting is associated with overweight in children of four nations that are undergoing the nutrition transition. Journal of Nutrition. 1996;126:3009-3016. 
17. Popkin B. The nutrition transition and its health implications in lower-income countries. Public Health Nutrition. 1998;1:5-22.

18. Hoffman DJ, Sawaya AL, Verreschi I, Tucker $\mathrm{KL}$, Roberts SB. Why are nutritionally stunted children at increased risk of obesity? Studies of metabolic rate and fat oxidation in shantytown children from São Paulo, Brazil. American Journal of Clinical Nutrition. 2000;72(3):702-707.

19. Schroeder DG, Martorell R, Flores R. Infant and child growth and fatness and fat distribution in Guatemalan adults. American Journal of Epidemiology. 1999;149:177-185.

20. Florencio T, Ferreira H, Tojal A, Cavalcante J, Sawaya A. Obesity and undernutrition in a very low income population in the city of Maceió, northeastern Brazil. British Journal of Nutrition. 2001;86:277-283.

21. Palloni A, McEniry M, Wong R, Pelaez $M$. The tide to come: Elderly health in Latin America and the Caribbean. Journal of Aging and Health. 2006;18(1):180-206.

22. Sicheiri R, Siquiera K, Moura A. Obesity and abdominal fatness associated with undernutrition early in life in a survey in Rio de Janeiro. International Journal of Obesity and Related Metabolic Disorders. 2000;24:614-618.

23. Barker D. Fetal and infant origins of adult disease. London: British Medical Journal Publishing; 1994.

24. Popkin B, Horton S, Soown K. The nutrition transition and prevention of diet-related diseases in Asia and the Pacific. Food and Nutrition BuIletin. 2001;22(Supl 4):S11-S21.

25. Popkin B. The nutrition transition and obesity in the developing world. Journal of Nutrition. 2001;131(3):871S-873S.

26. Linetzky B, De Maio F, Ferrante D, Konfino J, Boissonnet C. Sex-stratified socio-economic gradients in physical inactivity, obesity, and diabetes: evidence of short-term changes in Argentina. International Journal of Public Health. 2013;58(2):277-284.

27. De Maio FG, Linetsky B, Virgolini M. An average/deprivation/inequality (ADI) analysis of chronic disease outcomes and risk factors in Argentina. Population Health Metrics. 2009;7:8.

28. Fleischer NL, Diez Roux AV, Alazraqui $M$, Spinelli H, De Maio F. Socioeconomic gradients in chronic disease risk factors in middle-income countries: evidence of effect modification by urbanicity in Argentina. American Journal of Public Health. 2011;101(2):294-301.

29. Sohan AS, Ottenbacher KJ, Markides KS, Kuo YF, Eschbanch K, Goodwin JS. The effect of obesity on disability vs mortality in older Americans. Archives of Internal Medicine. 2007;167:774-780.

30. Vincent HK, Vincent KR. Obesity and inpatient rehabilitation outcomes following knee arthroplasty: A multicenter study. Obesity. 2008;16(1): 130-136.

31. World Health Organization. Obesity: preventing and managing the global epidemic (Technical Report Series 894). Geneva: World Health Organization; 2000.

32. Caballero B, Wang J. Commentary: obesity and mortality - light at the end but still a long tunnel. International Journal of Epidemiology. 2006;39:21-22.

33. Barrera G, Bunout D, Gatta's V, de la Maza MP, Leiva L, Hirsch S. A high body mass index protects against femoral neck osteoporosis in healthy elderly subjects. Nutrition. 2004;20(9):769-771.

34. Nakaoka D, Sugimoto T, Kaji H, Kanzawa M, Yano S, Yamauchi M, Sugishita T, Chihara K. Determinants of bone mineral density and spinal fracture risk in postmenopausal Japanese women. Osteoporos Int. 2001;12:548-555.

35. Wu F, Ames R, Clearwater J, Evans MC, Gamble G, Reid IR. Prospective 10-year study of the determinants of bone density and bone loss in normal postmenopausal women, including the effect of hormone replacement therapy. Clinical Endocrinology (Oxford). 2002;56(6):703-711.

36. Kato I, Toniolo P, Zeleniuch-Jacquotte A, Shore RE, Koenig KL, Akhmedkhanova A, Ribolic E. Diet, smoking and anthropometric indices and postmenopausal bone fractures: a prospective study. International Journal of Epidemiology. 2000;29(1):85-92.

37. Galvard H, Elmstahl S, Elmstahl B, Samuelsson SM, Robertsson E. Differences in body composition between female geriatric hip fracture patients and healthy controls: body fat is more important as explanatory factor for the fracture than body weight and lean body mass. Aging (Milano). 1996;8(4):282-286.

38. Albala C, Yañez M, Devoto E, et al. Obesity as a protective factor for postmenopausal osteoporosis. International Journal of Obesity and Related Metabolic Disorders. 1996;20(11):1027-1032. 
39. Karsenty G. Leptin controls bone formation through a hypothalamic relay. Recent Progress in Hormone Research. 2001;56:401-415.

40. Instituto Nacional de Estadística y Censos. Encuesta Nacional de Factores de Riesgo 2009: Documento para la utilización de la base de datos usuario [Internet]. 2010 [citado 14 feb 2014]. Disponible en: http://goo.gl/gBav9s.

41. World Health Organization. Obesity and overweight [Internet]. 2012 [citado 21 ago 2012]. Disponible en: http://goo.gl/ZiddVI.

42. World Health Organization. BMI classification [Interet]. 2006 [citado 1 nov 2012]. Disponible en: http://goo.gl/tVO4TY.

43. Correa MJC, Sierra LE. Intervalos de confianza para el parámetro de la distribución binomial. Revista Colombiana de Estadística. 2001;24(1):59-72.

44. Freedman DA. On the so-called "Huber Sandwich Estimator" and "Robust Standard Errors". The American Statistician. 2006;60(4):299-302.

45. Beckera J, Nora DB, Gomes I, Stringaria FF, Seitensusa R, Panossoa JS, Ehlers JAC. An evaluation of gender, obesity, age and diabetes mellitus as risk factors for carpal tunnel syndrome. Clinical Neurophysiology. 2002;113(9):1429-1434.

46. Schieman S, Turner HA. Age, disability, and the sense of mastery. Journal of Health and Social Behavior. 1998;39(3):169-186.

47. Monteverde $\mathrm{M}$, Noronha $\mathrm{K}$, Palloni A. Obesity and excess mortality among the elderly in the United States and Mexico. Demography. 2010;47(1):79-96.

48. Díaz-García J, González-Zapata LI, Estrada-Restrepo A. Comparación entre variables antropométricas y mediciones reales. Archivos Latinoamericanos de Nutrición. 2012;62(2):112-118.

49. Bender R, Jockel KH, Trautner C, Spraul M, Berger $M$. Effect of age on excess mortality in obesity. Journal of the American Medical Association. 1999;281:1498-1504.

50. Stevens J, Cai J, Pamuk E, Williamson D, Thun $M$, Word J. The effect of age on the association between body-mass index and mortality. New England Journal of Medicine. 1998;338:1-7.

\section{FORMA DE CITAR}

Monteverde M. Exceso de peso y discapacidad en las personas mayores de la Argentina. Salud Colectiva. 2015;11(4): 509-521.

Recibido: 1 de febrero de 2015 | Versión final: 17 de julio de 2015 | Aprobado: 20 de agosto de 2015

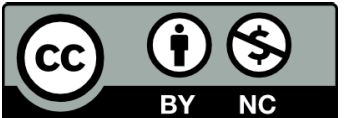

Este obra está bajo una licencia de Creative Commons Reconocimiento-NoComercial 4.0 Internacional. Reconocimiento - Permite copiar, distribuir y comunicar públicamente la obra. A cambio, se debe reconocer y citar al autor original. No Comercial - Esta obra no puede ser utilizada con finalidades comerciales, a menos que se obtenga el permiso. 